\title{
Texture Classification for Fake Indian Currency Detection
}

\author{
${ }^{1}$ P.Gayathri, ${ }^{2}$ P.Soniya, ${ }^{3} \mathrm{~V}$.YaminiPriya, ${ }^{4}$ G.Srinivas, ${ }^{5} \mathrm{~V}$.Ravindra \\ ${ }^{1} \mathrm{UG}$ Student, ${ }^{2} \mathrm{UG}$ Student, ${ }^{3} \mathrm{UG}$ Student, ${ }^{4} \mathrm{UG}$ Student, ${ }^{5}$ Associate professor \\ V.S.M.College of Engineering, Ramachandrapuram.
}

\begin{abstract}
In this paper, the automatic fake currency recognition system (AFCRS) is developed to identify the currency whether it is fraud or genuine. The present problems due to demonetization drags down the banking system and also in many areas. A newvision of curl neural network regarding of finding fake notes through their images is tested in this paper, which is relatively good than past image processing methods. This procedure depends upon intense study, which has seen enormous success in image segmentation charge in present times. This technique can help both human and electronics in finding a fake currency note in real time through an image of the same. The suggested method AFCRS can also be situated as an implementation in the android phone which can be more useful for common people for finding the real or fake note. The precise of the suggested system can be increased by the original fake notes, where as the suggested system has the images from children's bank churan label.
\end{abstract}

\section{INTRODUCTION}

Automatic identification of fake note is very crucial in some areas like banking, jewellery shops nowadays .This method is used to find whether the note is genuine or duplicate, by the automated system which is by convolution neural network, in intense learning. Intense learning shines in the task of identification and dividing the images over a huge data collection. The demonetization drive is a tramp to eradicate manipulation and black currency notes, but it badly fails to find the problems of counterfeit currency. A intense neural network is a mathematical model that works in a close trend to the neurons in our brain.Each neuron takes the data, undergo some functioning and give its result to the following neuron that is to its hidden layer. Recognizing a duplicate note by mere visual inspection is quite complex task. Most of the common people are not conscious of the safety features present in original currency notes and thus residue at risk.

\section{RESEARCH METHOD}

It is feasible for everyone to stamp fake notes with the help of computer and different scanning techniques. After demonetization this problem becomes more such

that it can be identified through many machines by various techniques listed below[10,7,1].

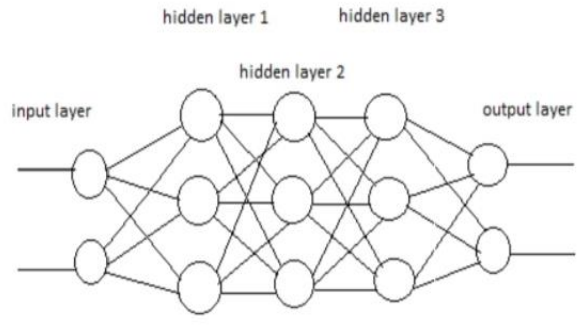

Figure1: A Deep neural network

\section{A. Techniques}

The sheet "ANN based currency identification system used squash gray scale and implementation for Sri Lankan notes"[9], handover by D.A.K.S Gunaratna, H.L and N.D. Kodikara. Premaratna developed a method of image data collection pre-processing through gray scale conversion, the image sub division, edge detection and PCA methods in the year 2008. The sheet "Bangladeshi currency note identification by neural network with centre line uniform marks"[14], invented by N.Jahangir, neural network identification scheme by back propagation algorithm using multi layer perceptron in the year 2007 was introduced by A.R.Chowdary. The paper "multiple kinds of paper currency identification using neural network and application for euro currency"[6] proposed by F.Takeda. T.Nishkage introduced the increase neurorecognition design using sensors in the year 2000. The review presented by A.Frosni, M.Gori and P.Priami in the year 1996 through a paper "A neural network model for paper identity and verification"[11] classifies and verifies through a model called multi- layered perceptron. The paper which is presented by F.Takeda, S.Omatu "High speed identification by neural networks(system)"[5] in the year 1995 tells that how the identification is done by an normal method of neural system that is among only one unseen layer, Input layer and output layer. The review held by M.Fukumi, N.Akamasu in the year 1996 by a paper "The method to design a neural system pattern identifies the system using a genetic algorithm with partial fitness and acceptance change"[15] uses the transmitted algorithm for identification system. These are few routine related to detecting and identifying in the fake currency paper.

\section{B. Software models for paper currency}

The method which is presented by mirza and nanda in the year 2012 tells that they take out 3 feature (identification mask, security band, and watermark) using edge based segmentation with the help of SOBEL operator [8].

In the year 2012, Sharma.J et.al proposed an algorithm based on LBP (local binary patterns) which produced good performance for image with low noise with $99 \%$ perfectly [2]. Mobile currency identification method using SIFT to identify partial images was proposed by Paisios et.al in the year 2012 states that the design is estimated using finite sample set with different state which are folded, imperfectly. They used KNN algorithm which has an accuracy $75 \%$ for the first method and 93\% for the second method.[4]

Sargano et.al initiate an algorithm feed forward back propagation neural network used for classification which 
consume less time when differentiate to other algorithms in the year 2013.[3]

In the year 2014 Da-Costa invented a bank note identification system to identify set of bank notes in a contrasting view and scales. He used feature detection, description and matching which are used to increase the belief in identifying results .But the suggested method is not acceptable for mobile phone because of high computation power. Whereas the outputs are vigorous to handle the folded and creased notes.[13]

Considering the raise compositions survey, we should consider an algorithm which grasp less time, keeps high precision, which can be adaptable for android phones and can also work with large data sets. Regarding to my observation, CNN algorithm is the one which convinces all the circumstances by omitting the restrictions which are in the above similar methods and papers. The advantage of $\mathrm{CNN}$ over the image processing method is that, $\mathrm{CNN}$ are exclusive type of neural network for processing input data that has an fundamental grid like topology. Generally, the data given to a $\mathrm{CNN}$ will have regular shape to it such that neighbouring are correlated.

A CNN strive to identify the most applicable patterns that help to find how to complete the given task. However the drawback of image processing is high in cost. CCD that is used for digital image processing has some drawbacks like memory card problems, higher cost and battery usage.

\section{III.THEORYAND EXPERIMENTATION}

In order to apply the proposed solution for finding fake notes, we reproduce the functions using $\mathrm{CNN}$ with the help of python language.

\section{A. Proposed solution}

we will be constructing a convolution neural network according to suggested algorithm which will be instruct on a given duplicate and real currency set, and later be able to forecast whether the currency is original or fake. In this AFCRS we will be decoding an image classification problem, where our aim will be to tell which category the input image belongs to. We are going in such a way to reach it is by teaching an unnatural neural network on image data set of currency and make the NN to forecast which class the image related to, when it sees an image having duplicate note or original note the next time. In CNN the feature extraction and classification takes place automatically within a single frame work. They have many advantages when compared to many other image processing methods.

\section{B. Architecture of $C N N$}

For our project we make use of python libraries like theano and tensor flow for applying and teaching the intense learning model. Many pre- trained models are available on the internet like Net, Alex Net, VGG Net, Google Net/Inception, and ResNet [12]. About VGG Net (figure 2), it is good because it contains of 16 layers with able to be learned. Parameters that is weights and biases. The pooling layers learned. To compress so many number of parameters in such very deep networks, in all convolution layer with the convolution stride set to ' 1 ' they used small $3 * 3$ filters. At the end of the network they are 3 fully connected layers. The VGG networks use multiple $3 * 3$ convolution layers to constitute complex attributes. Consequently, here we select VGG Net architecture ILSVRC
(Image Net Large Scale Visual Recognition Competition) 2014 competition

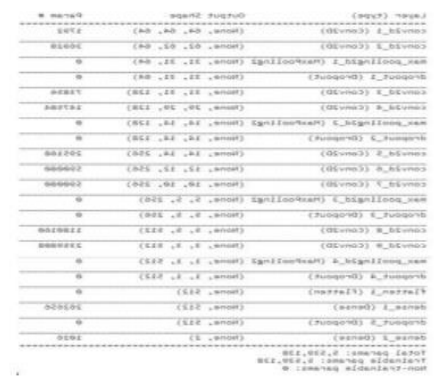

Figure 2: Output screenshot of VGG Net model summary generated from the experimental setup.

\section{Data-set}

We generated a data-set of paper currency notes. The data-set has been created for this particular resolution. The images have been gathered from different Google source. Data-set separated into 2 types. One is original another one is fake notes (figure 3 ). In this AFCRS we have used $75 \%$ of the images for the purpose of training and rest $25 \%$ of the images are used for testing. Then we allow the trained images as input to output model and train the model. In generating the data-set, we assumed that old currency notes which are used in advance demonetization as fake currency, as they are legally banned and if they are assumed as original currency, then it will become very hard while addressing the problems like in banking sectors.
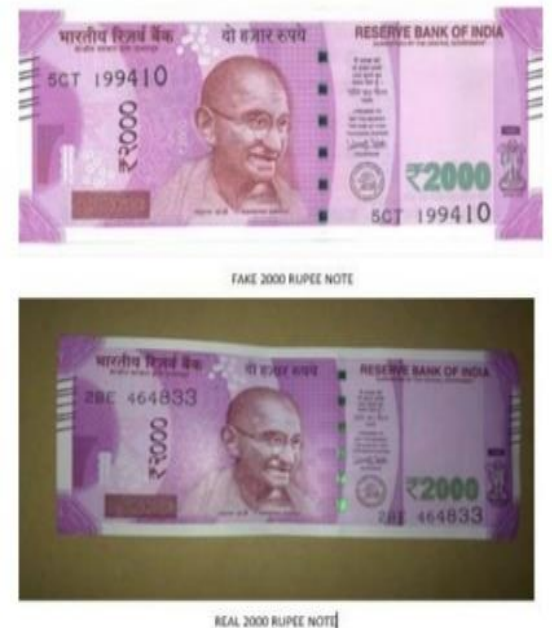

Figure 3: Fake and Original 2000 rupee note

\section{Methodology}

In coding role, use keras deep learning library in python to generate our CNN (Convolutional neural network). In this install the tensor flow is an open source software library. It is a representative math library and is also used for machine learning application such as neural networks. Theano is python library and improve for manipulating mathematical expressions.

In theano calculations are expressed using NumPy-esquare syntax and compiled to run productive on either CPU or GPU architectures.

Later installing the required libraries, we train output model as discussed above. Later training and testing the model, we 
adjust an epoch value which rise the accuracy of the AFCRS upon rise the value of epochs.

\section{Pre-processing}

The easy way to obtain the data without over-filtering and under-filtering is to pre-process the data-set. The major aim supporting the data pre-processing is to add the value to the base value which is data-set generated. The major lead of data pre-processing is to obtain a better training-set. For these causes, we use keras library for pre-processing the images. The VGG-16 model requires an input image shape of $64 * 64 * 3$, where 3 indicates to the $\mathrm{R}, \mathrm{G}, \mathrm{B}$ (red, green, blue) requirements of coloured image and the image must be $64 * 64$ pixel in size.

We then apply the following 3 types

a. Image Re-scaling

b. Image shearing

c. Perspective Transformations

a. Image Re-scaling

We require re-scaling the image to construct the model data in a standard format so that the training is better, correct and faster. We have re-scaling element in keras. To apply this element, we need to buy libraries from keras pre-processing as "Image data generator". If the re-scaling element is none or 0 , no re-scaling is applied, or else we multiple the data by the value provided. This process is finished after applying all other transformations. For output model AFCRS, we use a re-scaling element as: rescale $=1 / 255$ for training and testing data-set.

b. Image shearing

We need to shear the image to construct the training data better, exact. We also have shear range elements in keras. This is to be better from the keras pre-processing library.

For output model AFCRS, we use a sharing range "shearrange $=0.2 "$. Share range is shear angle in counter clockwise direction in degrees. It is called shear intensity.

c. Perspective transformations

The perspective transformations is applied on training data to zoom in the range "zoom-range $=0.2$ " to gain the correct results by study in correct manner. The zoom ranges are float or lower, upper ranges for random zoom. This is finished by importing a library from keras pre-processing.

2. Training the $\mathrm{CNN}$

Here, choose VGGNet for output model AFCRS. We finetuned the VGGNet [12]. Which is a pre-trained network? This speeds up the training process, since there are fewer layers to actually train. To train the neural network, it is important to start with a poor performing neural network and bring up the neural network with more perfection. In words of loss role, we want our loss role to be too much bottom in the end of training. This specifies our neural network has high study rate and perfection. The problem of training the network is similar to generate the loss function with lower error rate. It is main and well organized to reduce the loss because, it turns out that loss is much easier function to improve.

If there are so many algorithms that activation functions, we select ReLU (Rectifier linear unit) as our activation function, and we can also select "Adam" as our improve function because, it improves neural networks by high speed training and also computational step of ReLU is easy. To accept the given currency note as fake or real, we must see the perfect of the VGG-16 model fine-tuned with the generated data-set. It was suitable $55 \%$ on the corresponding test set, however our data-set was very little and limited to 200 images.

If we expand our image data-set through real-world trails can make the model high perfectly trained which may conclude our results beyond $80 \%$ perfect. This is quality sign for prediction of results. Despite the real score, the result must be assigned to over-fitting on the data-set, as the training and test set's are very equal. Therefore, later pre-processing we must examine the loss and perfect trends of our model, AFCRS by charging the batch. There are fundamentally two cases in deep studying regarding the loss and perfect values.

\section{IV.ACKNOWLEDGEMENTS}

I would like to thank my joint authors, Institute and Department for encouraging us through-out the project by helding project reviews and leading us through their precious imputations tirelessly

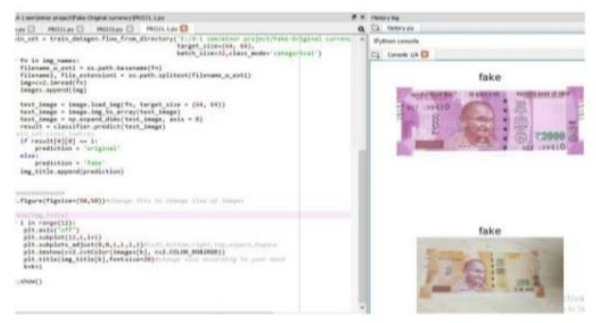

Figure 4: Screenshot of experimented model for recognizing fake currency.

\section{RECOGNITION OF CURRENCY}

After having the knowledge of fitted model, we now require identifying the note which is shown in the sample figures (figure $4 \& 5$ ), these are the screenshots of our test method, AFCRS. From this survey, it is distinctly see the difference between the genuine and fake currency note. So, it is therefore preferable to situate the same in mobile as an application which is a basic lead for the community.

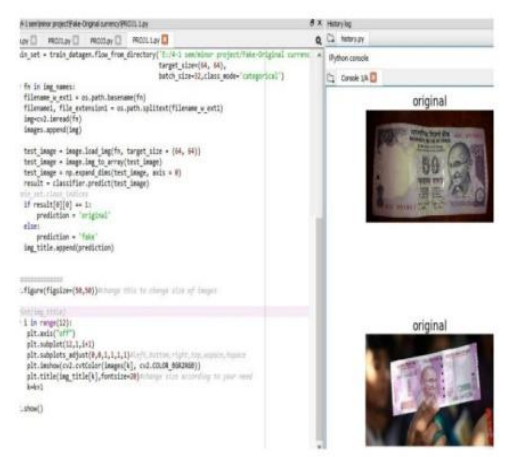

Figure 5: Screenshot of experimented model for recognizing original currency.

\section{CONCLUSION}

In this research, we suggest a procedure which explains the viability of using CNN with the VGG 16 architecture. As already the implemented data set was small and did not constitute the global scenario of duplicate currency data- set, it was useful till the whole experiment. The procedure of finding fake note using this model is more accurate in time and cost. From this we can also convince that receiving the original and 
large data-set, the model AFCRS can be subservient and also provide correct outputs, which can be more useful for the people in identifying the money whether it is duplicate or original.

Later analysis in this model can develop an android app which makes the world more agreeable in finding fake notes. This process can also be differentiate with many architectures of $\mathrm{CNN}$, which may have minor error rate than the existing model and can be merge by applying image pre-processing methods like edge-detection to cut the unnecessary surroundings of the note out of an image which results in effective outputs.

\section{REFERENCES}

[1] V.Vashishtha, M.Sadim, A paper currency Recognition system using Image processing to Improve the Reliability with PCA method, International Journal of Engineering Science \& (2015).

[2] J.S. Dewan, Extended Local Binary Pattern for Face Recognition, Technology ICAET, 2015.

[3] M. Sarfraz, A. Sargano, Robust Features and Paper Currency Recognition, in: The 6th International Conference on Information Technology Cite this publication.

[4] Paisios, Currency recognition using a smart phone: Comparison between colour SIFTS and gray scale SIFT algorithms (2012).

[5] S. Omatu, F. Takeda, High speed paper currency recognition by neural networks (1995) 73-77.

[6] T. Nishikage, F. Takeda, Multiple kinds of paper currency recognition using neural network and application for euro currency (2000) 27-47.

[7] P.R. Nagpure, T. Ghotkar, S. Shetty, Recognition and Fake Note Detection, International Journal of Innovative Research in Computer and communication Engineering vol 4 (March 2016).

[8] R. Mirza, V. Nanda, Design and Implementation of Indian Paper Currency Authentication System Based on Feature Extraction by Edge Based Segmentation Using Sobel, Operator, International Journal of Engineering Research and Development 3 (2012) 41-46.

[9] D.A.K.S. Gunaratna, N.D.Kodikara, Premaratne, ANN Based Currency Recognition System using Compressed Gray Scale and Application for Sri Lankan Currency Notes - SLCRec, World Academy of Science, Engineering and Technology International Journal of Computer and Information Engineering 2 (2008) 29572962.

[10] P. Gidveer, S.R. Darade, Automatic Recognition of Fake Indian Currency Note, International Conference on Electrical Power and Energy Systems (ICEPES), Bopal, India, pp.290-294.

[11] P.P.A. Frosini, A neural network based model for paper currency recognition and verification, IEEE Transactions on Neural Networks 7 (1996).1482-1490.

[12] S.Das, CNN Architectures: LeNet, AlexNet, VGG, GoogLeNet, ResNet and more, 2017

[13] Da-costa, Multiview banknote recognition with component and shape analysis (2014).

[14] A.Chowdhury, N.Jahangir, Bangladeshi banknote recognition by neural network with axis symmetrical masks in masks, in: 10th international conference on computer and information technology.

[15] N. Akamatsu, M. Fukumi, A method to design a neural pattern recognition system by using a genetic algorithm with partial fitness and a deterministic mutation (1996)

\section{AUTHOR PROFILE}

P.Gayathri, Student, Bachelor of Technology, Electronics \& Communication Engineering at V.S.M College of Engineering, Ramachandrapuram.

P.Soniya, Student, Bachelor of Technology, Electronics \& Communication Engineering at V.S.M College of Engineering, Ramachandrapuram.

V.YaminiPriya, Student, Bachelor of Technology, Electronics \& Communication Engineering at V.S.M College of Engineering, Ramachandrapuram.

G.Srinivas, Student, Bachelor of Technology, Electronics \& Communication Engineering at V.S.M College of Engineering, Ramachandrapuram.

V.Ravindra, Associate Professor. Electronics \& communication Engineering at V.S.M College of Engineering Ramachandrapuram, E.G.District. His area of interest includes communication systems and wireless communication. 